\title{
Cross-linking and charging molecular magnetoelectronics
}

Yulong Huang, ${ }^{\dagger}, *$ Yuxuan Chen, ${ }^{\S}$ Yong Hu,${ }^{\dagger}$ Travis Mitchell, ${ }^{\ddagger}$ Lu An, ${ }^{\dagger}$ Zheng Li, ${ }^{\dagger}$ Jason Benedict, ${ }^{\dagger}$ Huashan $\mathrm{Li}^{\S}, *$ and Shenqiang $\operatorname{Ren}^{\dagger, \dagger, \perp, *}$

${ }^{\dagger}$ Department of Mechanical and Aerospace Engineering, University at Buffalo, The State University of New York, Buffalo, NY, 14260, USA

${ }^{\S}$ School of Physics, Sun Yat-Sen University, Guangzhou 510275, China

Department of Chemistry, University at Buffalo, The State University of New York, Buffalo, NY, 14260, USA

${ }^{\perp}$ Research and Education in energy, Environment and Water (RENEW) Institute, University at Buffalo, The State University of New York, Buffalo, NY, 14260, USA.

*E-mail: yhuang59@buffalo.edu; lihsh25@mail.sysu.edu.cn; shenren@buffalo.edu 


\section{Table of Contents}

1. MC composites with different concentrations $p$ and $T_{\text {c. }}$

2. The surface morphologies of MCs

3. The first-principle calculations for cross-linking structure.

4. UV-Vis-NIR spectroscopy

5. The Raman spectra of MCs.

6. X-ray diffraction patterns of all MCs.

7. Thermal analysis of MCs.

8. Magnetic properties of MCs.

9. DFT calculations of surface magnetic moment in MCs.

10. Conductivities and hopping mode fitting of MCs.

11. Absorbance spectra of MCs under electric field.

12. Temperature dependent magnetization of pure PBA under electric field.

\section{METHODS}

Sample preparation: $\operatorname{Poly}(3,4-$-ethylenedioxythiophene) polystyrene sulfonate (PEDOT:PSS) in aqueous solution with $\mathrm{pH}=2 \sim 3.5$, vanadium(II) chloride $\left(\mathrm{VCl}_{2}\right)$ and potassium hexacyanochromate(III) $\left(\mathrm{K}_{3} \mathrm{Cr}[\mathrm{CN}]_{6}\right)$ were used as purchased from Sigma-Aldrich. $72.2 \mathrm{mg}$ $\mathrm{K}_{3} \mathrm{Cr}[\mathrm{CN}]_{6}$ was dissolved by $20 \mathrm{ml}$ deionized water in a $50 \mathrm{ml}$ centrifuge tube, followed by adding PEDOT:PSS solution in different weight ratios compared to $\mathrm{K}_{3} \mathrm{Cr}[\mathrm{CN}]_{6}$. 5-minutes ultrasonication was applied to get an uniform $\mathrm{K}_{3} \mathrm{Cr}[\mathrm{CN}]_{6}$ and PEDOT:PSS solution. Then, 48.2 mg $\mathrm{VCl}_{2}$ was added into above solution, and the centrifuge tube was fully filled by adding enough deionized water again. The well sealed centrifuge tube was placed under ultrasonication for 1 hour, and then was aged for 12 hours in a vacuum chamber. After that, dark blue participates of Prussian blue analogue and PEDOT:PSS composites (MCs) were collected by 
centrifugation, washing using deionized water for 3 cycles. Finally, the MCs powder was dried in a vacuum drying oven for 12 hours.

Structural and morphologic characterizations: X-ray diffraction measurements were carried on the Rigaku Ultima IV $(40 \mathrm{kV}, 44 \mathrm{~mA})$ to characterize the structure of cross-linking MCs powder. Carl Zeiss AURIGA (200 kV) Field Emission Scanning Electron Microscope (FESEM) with Oxford Energy-dispersive X-ray Spectrometer (EDS) was used to collect the surface morphology and element ratio $p$ of MCs composites obtained under different PEDOT:PSS concentrations.

Spectroscopy measurements: UV-Vis-NIR spectra of MCs and their synthetic process were collected on Agilent Cary 7000 universal measurement spectrophotometer. For visible absorption measurements under electric field, MCs power suspended in water was dropped onto a glass microscope slide on which an electrode pattern with a gap of $153 \mu \mathrm{m}$ confined MCs powder. Then, sample was dried and examined using an inverted microscope (Olympus IMT2). When electric field was applied, spectra were recorded using a diffraction grating (150 g $\mathrm{mm}^{-1}$ ) by dispersing a single line of the image. An intensified charge-coupled-device Camera (PIMAX3, Princeton Instruments, Intensifier, Unigen II, $1024 \times 256$ ) recorded the light intensity. Commercial software (Princeton Instruments, Lightfield ver. 6.7.1804) was used to calibrate wavelength and acquire data. Fourier Transform Infrared Spectra measurements were conducted on Bruker VERTEX 70 spectrometer with attenuated total reflection mode. All the Raman measurements were conducted on Renishaw inVia Raman Microscope with a laser wavelength of $514 \mathrm{~nm}$.

Computational methods: The first-principles calculations were performed using the planewave pseudopotential as implemented in the Vienna ab initio simulation package (VASP) ${ }^{[1]}{ }^{[2]}$ code. The electron-core interactions were described with frozen-core projector-augmented 
wave (PAW) pseudopotentials. The generalized gradient approximation (GGA) ${ }^{[3]}$ with the Perdew-burke-Ernzerhof (PBE) parameterization method was used as the exchange-correlation functional. Herein. we set a cut-off energy of $400 \mathrm{eV}$ to ensure the desired accuracy in all of our calculations. A reciprocal space sampling with a $\Gamma$-centered $1 \times 2 \times 1 \mathrm{k}$-points mesh was set in the Brillouin zone. The total energy convergence criteria was $1.0 \times 10^{-5} \mathrm{eV}$ and the force on each atom converged to $0.1 \mathrm{eV} / \AA ̊$ while optimizing the geometric structure. The van der Waals $(\mathrm{vdW})$ interaction was considered using the DFT-D3 with the Becke and Johnson(BJ)-damped method of Grimme ${ }^{[4]}$ and the dipole correction was employed. In addition, the vacuum region was set to $15 \AA$ along the $Z$ direction to avoid periodic images. DFT (GGA)+U within Dudarev's approach ${ }^{[5]}$ was used to draw reasonable conclusions. A U value of $4.5 \mathrm{eV}$ and 3.5 eV was applied for the V 3d states and Cr 3d states, respectively.

Magnetic susceptibility and hysteresis loop measurements. Magnetic properties measurements were conducted on a Vibrating Sample Magnetometer (VSM, MicroSense EZ7$380 \mathrm{~V})$ equipped with liquid nitrogen as cooling source. After cooling at zero magnetic field, magnetization under 10 Oe was measured from $110 \mathrm{~K}$ to $340 \mathrm{~K}$. Magnetic field dependent magnetization was measured at fixed temperatures by sweeping the magnetic field from $5 \mathrm{kOe}$ to $-5 \mathrm{kOe}$. The magnetization under bias voltage was measured in the same VSM, for which Keithley 2450 was used to supply voltage source and collect resistance.

Low temperature electric transport measurements. The standard four-probe method was used to measure the temperature dependent conductivity of MCs on a Janis low temperature system with helium gas compressor (CTI-Cryogenics, Helix Technology Corp.) from $300 \mathrm{~K}$ to $10 \mathrm{~K}$. The laser source was achieved from a fiber coupled blue-violet laser (405 nm, $0.5 \mathrm{~W})$ which is $100 \mathrm{~mW} / \mathrm{cm}^{2}$. All MCs for conductivity measurements were pressed into pellets before tests. 


\section{MCs with different concentrations $p$ and $T_{\text {c }}$.}

The high- $T_{\mathrm{c}}$ molecular magnetic compounds (MCs) with different concentration $p$ (atomic ratio of sulfur and vanadium) were synthesized in the same method as discussed in the method section of the maintext. The concentration of PEDOT:PSS is controlled by weighing different mass compared to the starting material $\mathrm{K}_{3} \mathrm{Cr}(\mathrm{CN})_{6}$. As inceasing the weight of PEDOT:PSS in the mixed solitution of PEDOT:PSS and $\mathrm{K}_{3} \mathrm{Cr}(\mathrm{CN})_{6}$, the synthesized MCs present variable magnetic ordering temperature $\left(T_{\mathrm{c}}\right)$ and an increasing detected concentration $p$ by energydispersive X-ray spectroscopy (EDS) (Table S1).

Table S1. High-Tc molecular MCs with different concentrations $p$ and $T_{\mathrm{c}}$.

\begin{tabular}{|c|c|c|c|c|c|c|c|}
\hline Sample & $\# 30$ & $\# 18$ & $\# 5$ & $\# 10$ & $\# 12$ & $\# 14$ & $\# 15$ \\
\hline Weight $(\%)^{\mathrm{a}}$ & 0 & $2 \%$ & $10 \%$ & $30 \%$ & $50 \%$ & $70 \%$ & $100 \%$ \\
\hline$p^{\mathrm{b}}$ & 0 & 0.07 & 0.16 & 0.37 & 0.77 & 0.88 & 1.21 \\
\hline$T_{\mathrm{c}}$ & $320 \mathrm{~K}$ & $337 \mathrm{~K}$ & $334 \mathrm{~K}$ & $285 \mathrm{~K}$ & $243 \mathrm{~K}$ & - & - \\
\hline
\end{tabular}

a: the weight ratio of PEDOT:PSS and $\mathrm{K}_{3} \mathrm{Cr}(\mathrm{CN})_{6}$,

${ }^{\mathrm{b}}: p=$ atomic ratio of sulfur $(\mathrm{S})$ and vanadium $(\mathrm{V})$ 


\section{The surface morphologies of MCs.}

The scanning electron microscopic images are taken on the dried precipitates of MCs, exhibiting the evolution of macro-structures with the concentration $p$. The pure V-Cr PBA $(p=0)$ shows an amorphous surface of clusters which is common in those MCs of $p=0.16$ and 0.37 . However, in the optimized MC with a concentration of $p=0.07$, it looks like more well defined in shapes than those of $p=0,0.16$ and 0.37 . As increasing the concentration of PEDOT:PSS ( $p=0.77,0.88$, and 1.21), the surface morphologies of MCs are in ribbon shapes as a consequence of polymer chains.

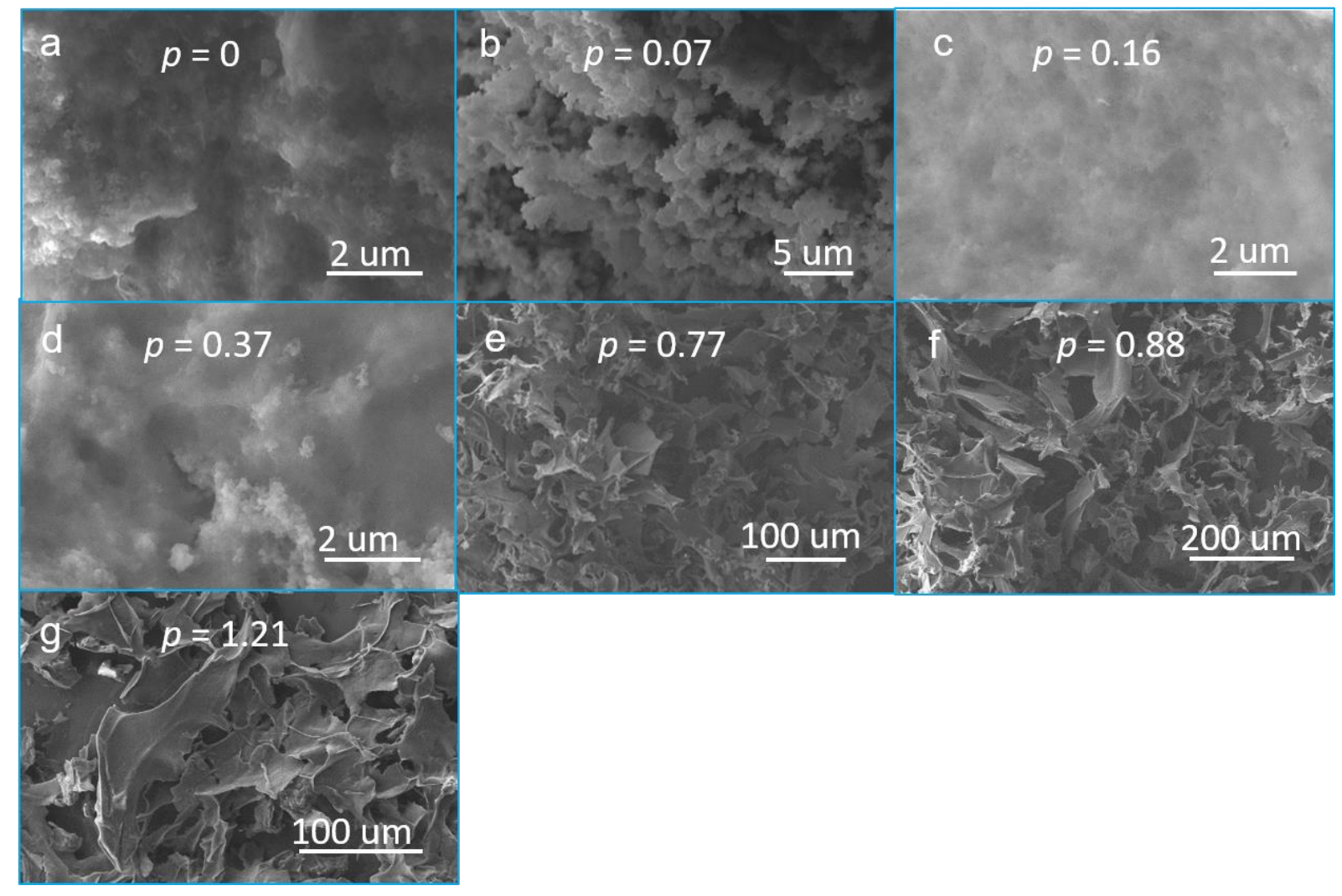

Figure S1. The surface morphologies of MCs are modified with the PEDOT:PSS concentration (a) $p=0$; (b) $p=0.07$; (c) $p=0.16$; (d) $p=0.37$; (e) $p=0.77$; (f) $p=0.88$; (g) $p=1.21$. 


\section{The first-principle calculations for cross-linking structure.}

We stated the cross-linking originates from the interaction between $\mathrm{SO}^{3-}$ of PSS and $\mathrm{V}$ according to the spectra evidence of pronounced V-O bond (Figure 2) and additional effects on other bonds (like $\mathrm{O}=\mathrm{S}=\mathrm{O}$ ) in both PSS and PBA (Figure $\mathrm{S} 4$ and S5). Such strong evidence from spectra supports the cross-linking structure between $\mathrm{SO}^{3-}$ of PSS and V cation. Besides, firstprinciple calculations confirm the stable cross-linking structure in energy between PSS and V cation.

However, we would not completely deny the possibility of the reaction between PEDOT with $\mathrm{V}$ cation since spectra signature of PEDOT also develop a little after in-situ cross-linking reaction. But structure and charge feature of PEDOT and V-Cr PBA allow us to consider the reaction between PEDOT with V cation is not popular. First, the co-planar structure of PEDOT polymers defines a close distance of adjacent oxygen atoms and forms a small inner circle of --C-O-CCC-S-CCC-O--- as marked by red color in below Figure S2g. The space of an inner circle is too limited to allow the reaction $\mathrm{V}$ cation with $\mathrm{O}$ in the inner circle, not allowing $\mathrm{V}-\mathrm{Cr}$ PBA structure to stay in. One possibility is that $\mathrm{V}$ cation reacts with a monomer at the end of a PEDOT chain as a simplified calculation in Figure S2d and S2e, resulting a small amount of cross-linking. The structure of $\mathrm{SO}^{3-}$ in PSS possesses a high flexibility in freedom degrees because benzenesulfonate $\left(\mathrm{C}_{6} \mathrm{H}_{5}-\mathrm{SO}_{3}{ }^{-}\right)$is conjunct onto a carbon chain by a single $\mathrm{C}-\mathrm{C}$ bond. Besides, $\mathrm{SO}^{3-}$ anion in PSS with negative charges is more active to react with $\mathrm{V}$ cation in PBA, while PEDOT carries positive charges that repels $\mathrm{V}$ cation. Based on above analysis, the reaction between $\mathrm{SO}^{3-}$ in PSS and $\mathrm{V}$ cation is dominant, while the combination of PEDOT and $\mathrm{V}$ cation is very limited regarding of structural confinement and charge interaction. Thus, we stated the cross-linking structure originates from the interaction between $\mathrm{SO}^{3-}$ of PSS and V cation. 

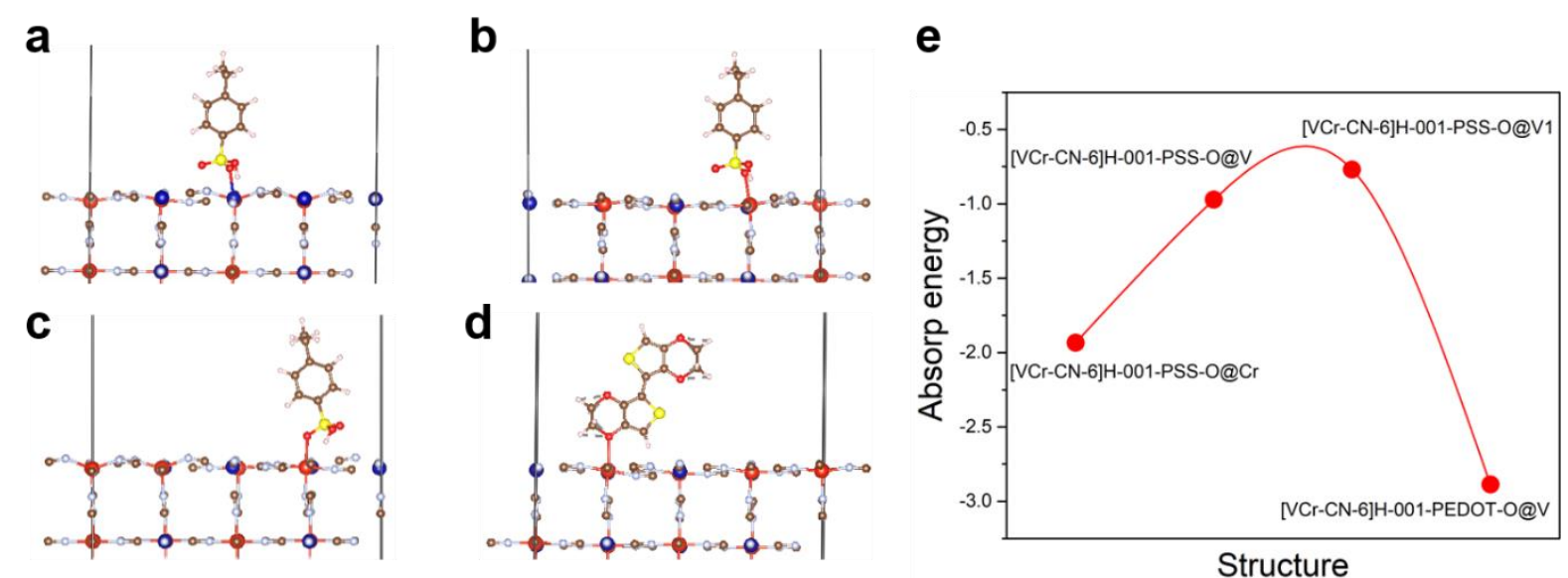

f PSS carries negative charges<smiles>CC(C)C(CC(CC(CC(CC(C)(C)C)c1ccc(S(=O)(=O)O)cc1)c1ccc(S(=O)(=O)O)cc1)c1ccc(S(=O)(=O)O)cc1)c1ccc(S(=O)(=O)O)cc1</smiles>

g PEDOT carries positive charges<smiles>CC(C)(C)SC1=C2OCCOC2C(C)(C)[SH]1c1sc(-c2sc(-c3sc(C(F)(F)F)c4c3OCCO4)c3c2OCCO3)c2c1OCCO2</smiles>

Figure S2. The first-principles calculations for cross-linking structures in MCs. The optimized results show the coordinations when the oxygen atom in $\mathrm{SO}_{3}{ }^{-}$anion approaches to (a) $\mathrm{Cr}$, (b) $\mathrm{V}$, (c) the center of $\mathrm{SO}_{3}{ }^{-}$anion approaches to $\mathrm{V}$, and (d) when oxygen atom in PEDOT approaches to V. (e) The absorpance energy for those coordinations indicate the cross-linking between $\mathrm{SO}_{3}{ }^{-}$anion and $\mathrm{V}$ cation is preferred. The calculation for PEDOT approaching $\mathrm{V}$ is not selected due to too much simplification of long-chain PEDOT as a monomer. (f-g) Structure of PSS and PEDOT. 


\section{UV-Vis-NIR spectroscopy}
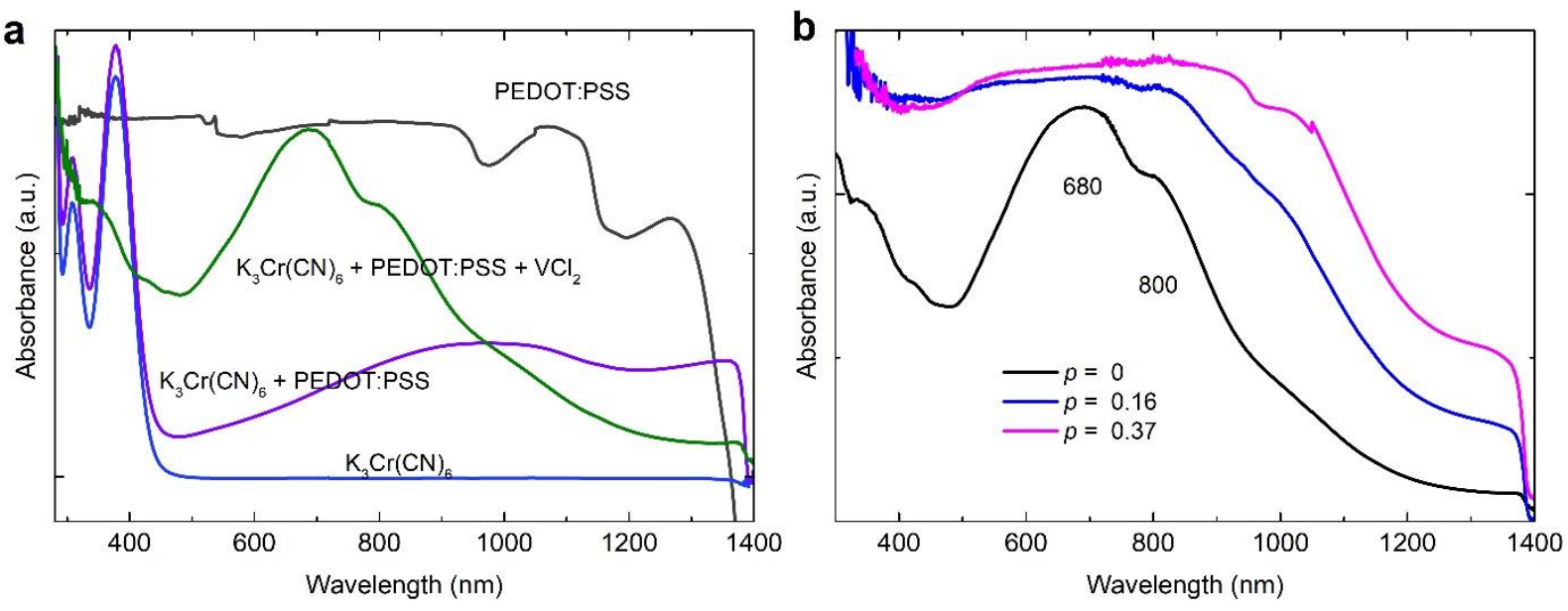

Figure S3. UV-Vis-NIR, FTIR, and Raman spectra of V-Cr based PBA, PEDOT:PSS and synthesized MCs. (a) UV-Vis-NIR spectroscopy of starting materials $\mathrm{K}_{3} \mathrm{Cr}(\mathrm{CN})_{6}$, PEDOT:PSS and synthesized MCs with $p(\mathrm{~S} / \mathrm{V})=0.07$ show the absorbance difference during the synergistic process. (b) UV-Vis-NIR spectroscopy of MCs with $\mathrm{p}=0,0.16$ and 0.37 exhibit the enhanced absorbance from $400 \mathrm{~nm}$ to $1400 \mathrm{~nm}$ by PEDOT:PSS.

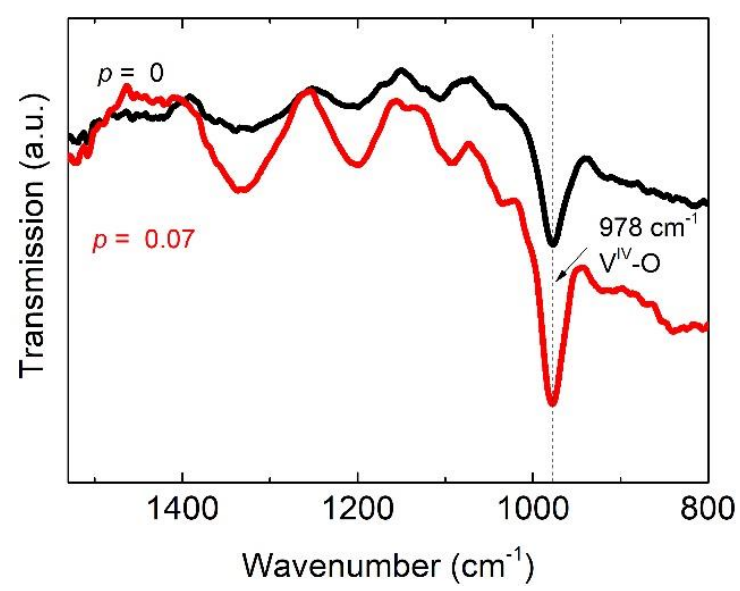

Figure S4. Enhancement of V-O vibration mode shows the cross-linking between PEDOT:PSS $(p=0.07)$ and $\operatorname{PBA}(p=0)$. 


\section{Raman spectra of MCs.}

Raman spectroscopy is often used to determine the vibrational modes of molecules, which is very helpful to detect the electronic fingerprints of chemical bonds. In the cross-linked $\mathrm{MCs}$, the chemical bonds may vary in the vibration frequencies as well as present new vibration modes.
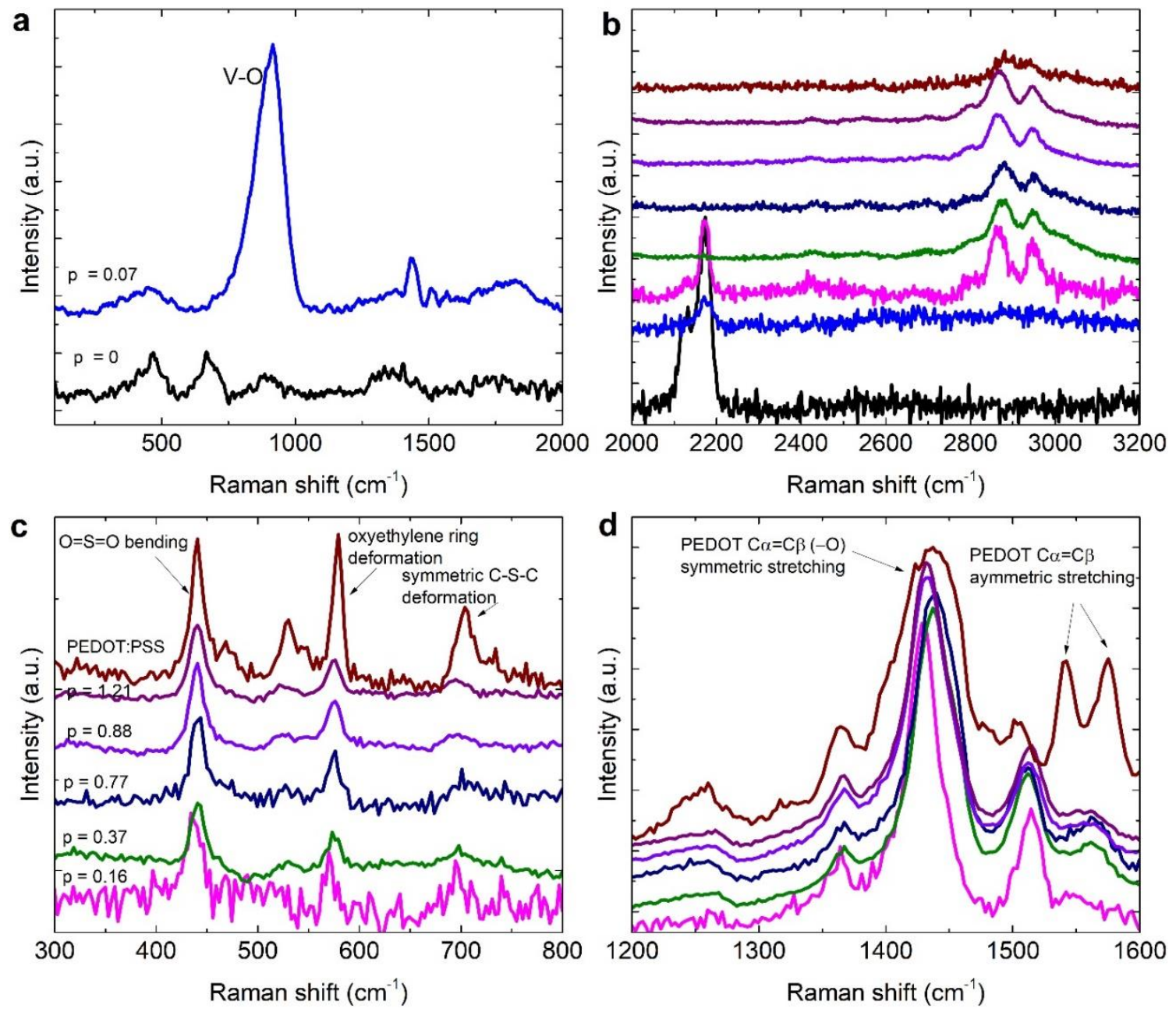

Figure S5. The Raman spectra of MC composites with different $p$. (a) Raman spectra of MCs indicate the V-O vibration enhancement at $915 \mathrm{~cm}^{-1}$ by cross-linking. (c) The evolution of Raman vibration modes of $\mathrm{O}=\mathrm{S}=\mathrm{O}$ bending, oxyethylene ring deformation, and symmetric $\mathrm{C}$ S-C deformation from PEDOT:PSS reflect the influence of cross-linking. (d) The symmetric and asymmetric stretching. 


\section{The X-ray diffraction patterns of MCs.}

The X-ray diffraction (XRD) patterns of MCs can tell the crystallinity, even though the pure V-Cr PBA often shows weak and broad peaks. For $p=0.07$ and 0.16 , the XRD patterns are not changed much while three unknown sharp peaks arise. The three peaks continue to present until $p=1.21$. At the meantime, the weak peaks of $\mathrm{V}-\mathrm{Cr}$ PBA is not visible when $p>0.37$, implying only an appropriate concentration of PEDOT:PSS can stabilize V-Cr PBA. For the highest concentration of $p=1.21$, the XRD pattern shows only a broad zone around $20^{\circ}$ which is different from others. The gradually faded XRD pattern of V-Cr PBA when $p>0.37$ indicates the decreasing crystallinity or a small size of cluster. The polymers PEDOT:PSS has not shown specific peaks on XRD patterns of MCs.

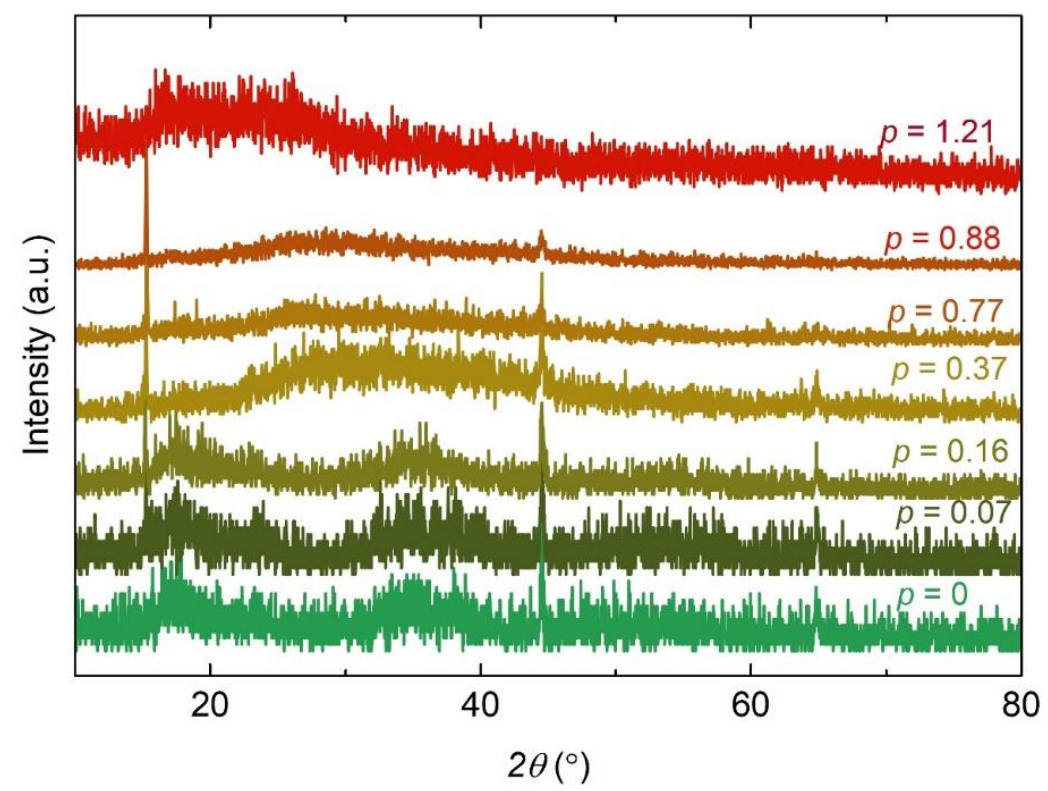

Figure S6. X-ray diffraction patterns of all MCs. 


\section{Thermal gravimetric analysis of MCs.}

The thermal gravimetric analysis (TGA) is applied to know the relative mass ratio of the components in MCs. It's noticed that the loss of water around $400 \mathrm{~K}$ is not significant in pure PBA $(p=0)$, while becomes almost $20 \%$ in the MC with $p=0.37$. With increasing the concentration $p$, MCs bear more water in those structures. From the weight vs. temperature curves, the tendency of weight loss in $\mathrm{MC}$ with $\mathrm{p}=0.07$ is the middle state to bridge PBA and MCs with a higher concentration.

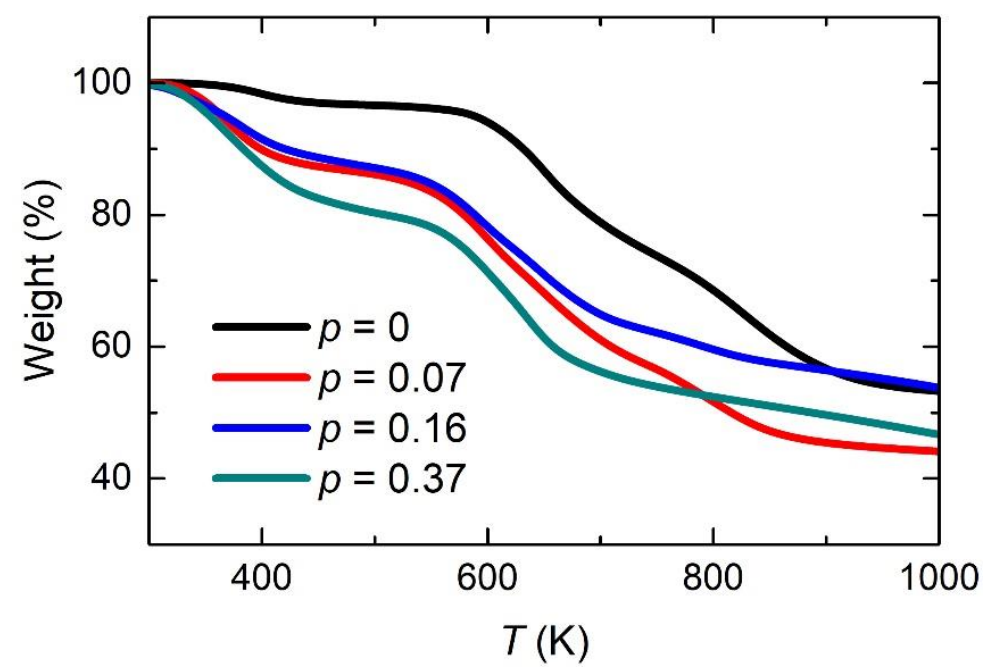

Figure S7. TGA of MCs for $\mathrm{p}=0,0.07,0.16$ and 0.37 . 


\section{Magnetic properties of MCs}

The magnetic properties of MCs with $p=0.88$ and 1.21 are not directly following others with a lower concentration $p$. The magnetization of MC with $p=0.88$ is almost three magnitude order lower than those with a lower $p$. Interestingly, the temperature dependent magnetization exhibits a very broad plateau between $150 \mathrm{~K}$ and $250 \mathrm{~K}$, and then decreases at lower temperature. Besides, a strong diamagnetic background is dominant at a high magnetic field that is obvious in the magnetic field dependent magnetization. But this MC still shows a ferrimagnetic feature with $T_{\mathrm{c}}$ around $325 \mathrm{~K}$ and a small magnetic loop in despite of much low magnetization. For the MC with $p=1.21$, the magnetization shows a plateau above $230 \mathrm{~K}$, but rapidly goes up at low temperature and also present a strong dimagnetic background at a higher magnetic field.
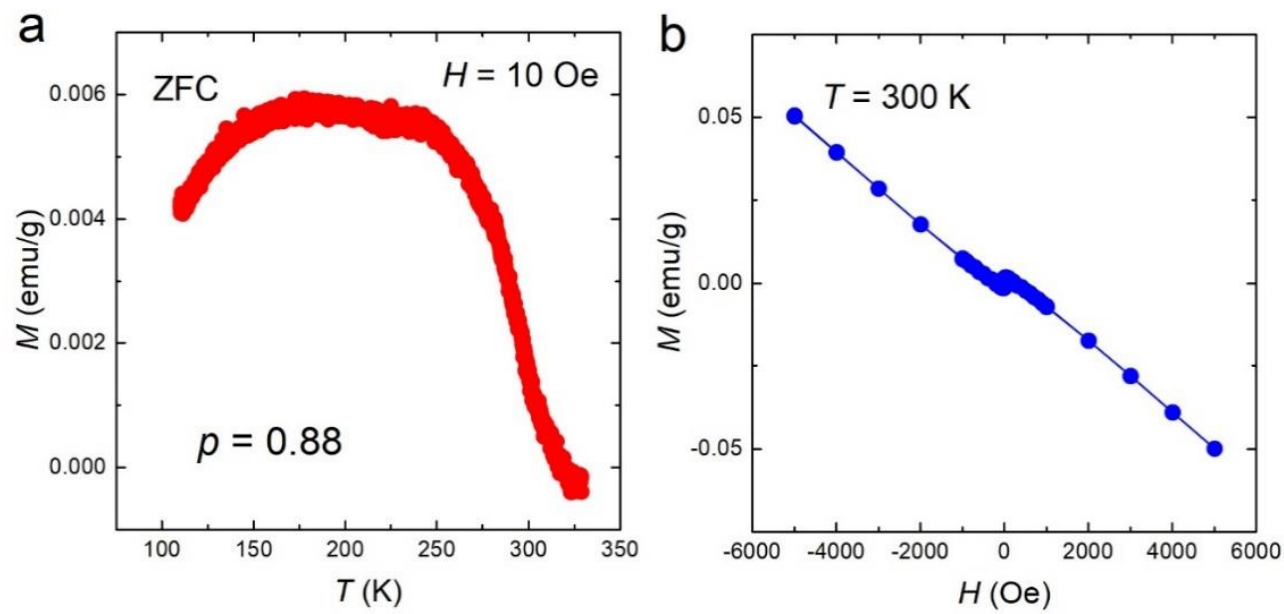

Figure S8. Magnetic properties of $M C$ with $p=88$. (a) Temperature dependent magnetization under 10 Oe by zero-field-cooling; (b) Magnetic field dependent magnetization at $300 \mathrm{~K}$. 

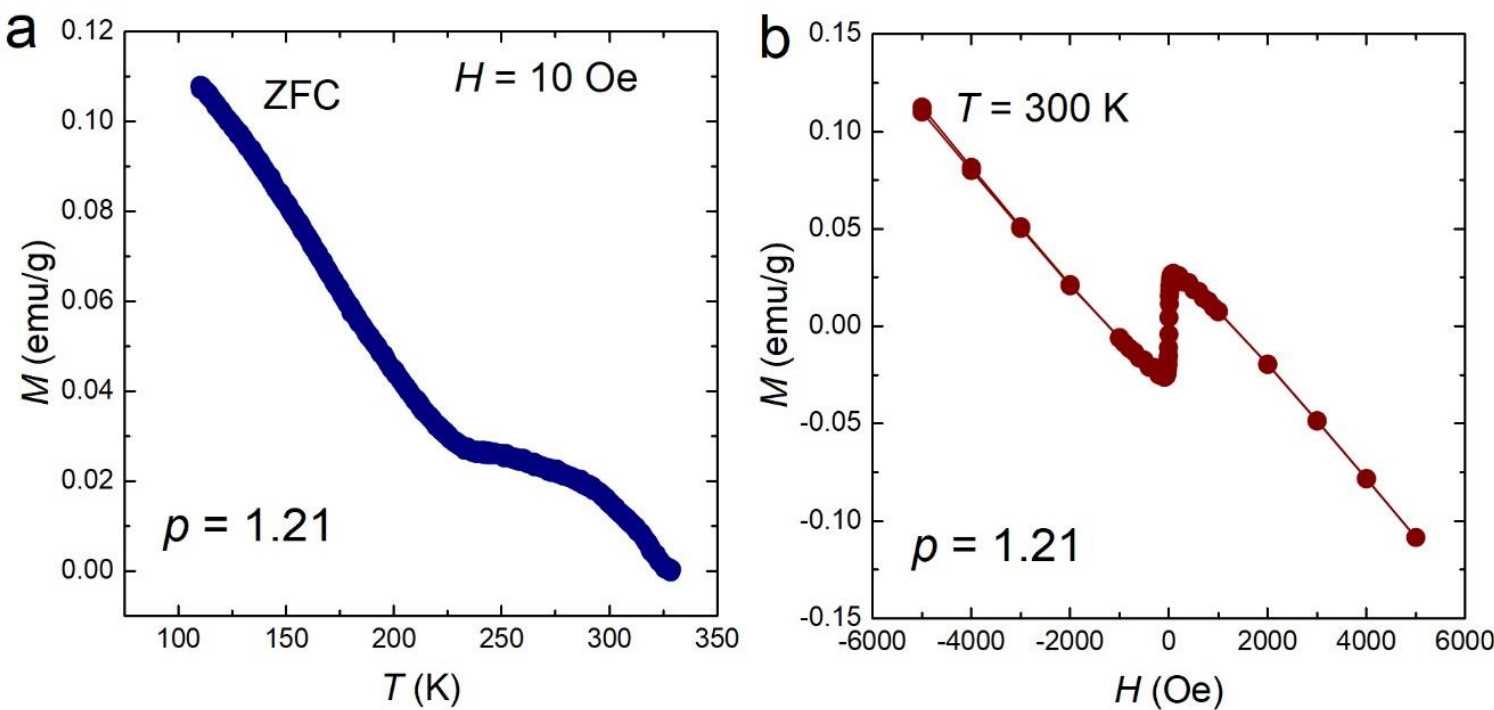

Figure S9. Magnetic properties of MC with $\mathrm{p}=1.21$. (a) Temperature dependent magnetization under 10 Oe by zero-field-cooling; (b) Magnetic field dependent magnetization at $300 \mathrm{~K}$. 


\section{DFT calculations of surface magnetic moment in MCs.}

The cross-linking between $\mathrm{SO}_{3}{ }^{-}$anion and $\mathrm{V}$ cation (Figure 1b) is preferred among many structural models based on the adsorption energy calculations (Figure S2). The calculated OV bond length at the interface is $2.24 \AA$, which is in agreement with previous theoretical studies ${ }^{[6 \text {, }}$ 7]. To understand how the cross-linking affects the magnetism of V-Cr PBA, we performed DFT calculations on various spin configurations to identify the spin ordering of bulk and surface systems. Consistent with reported studies, the results suggest that V-Cr PBA is a ferrimagnetic material with opposite magnetic moments between the $\mathrm{Cr}$ and $\mathrm{V}$ atoms coupled via the superexchange magnetism. The $\left[\operatorname{VCr}(\mathrm{CN})_{6}\right](001)$ surface is modeled by a slab with three $\mathrm{V}-\mathrm{Cr}$ atomic layers. The spin ordering of the bottom two layers is similar to that of the bulk system, while the presence of dangling bonds leads to the reversal of magnetic moments at the top layer (Figure S10). With the PSS adsorbed at the V-Cr PBA surface, the magnetic moments of the atoms at the top layer return to be aligned with those at the bottom two layers (Figure S11). This may be ascribed to the substantial change of electronic structures, as seen from the shift of Fermi level and the appearance of new surface states associated with the OV bond within the bandgap (Figure S11). The predicted rise of total magnetism of $\left[\operatorname{VCr}(\mathrm{CN})_{6}\right](001)$ slab from 9.652 $\mu_{\mathrm{B}}$ to $15.498 \mu_{\mathrm{B}}$ after PSS adsorption is consistent with the rise of magnetism with increasing PSS density at low concentration observed in experiment.

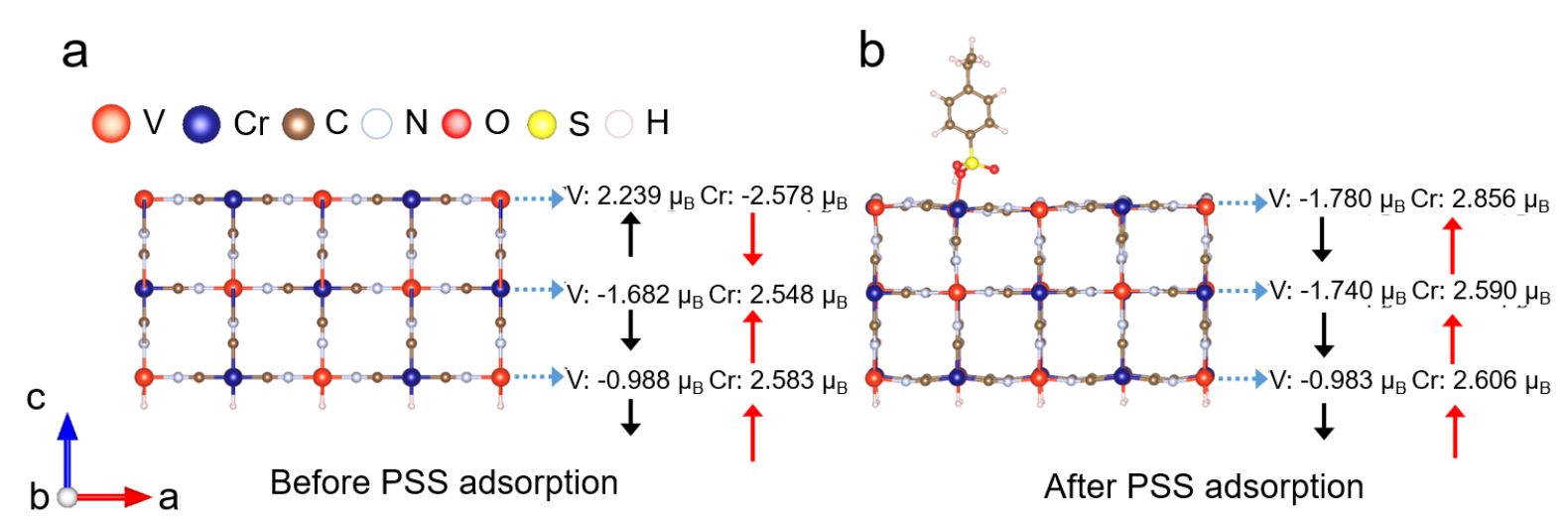


Figure S10. Atomic structure of (a) $\left[\mathrm{VCr}(\mathrm{CN})_{6}\right](001)$ surface, (b) $\left[\operatorname{VCr}(\mathrm{CN})_{6}\right](001)$-PSS

interface. The average magnetic moments of the same element in the same layer are presented on the right side of the structures.

a

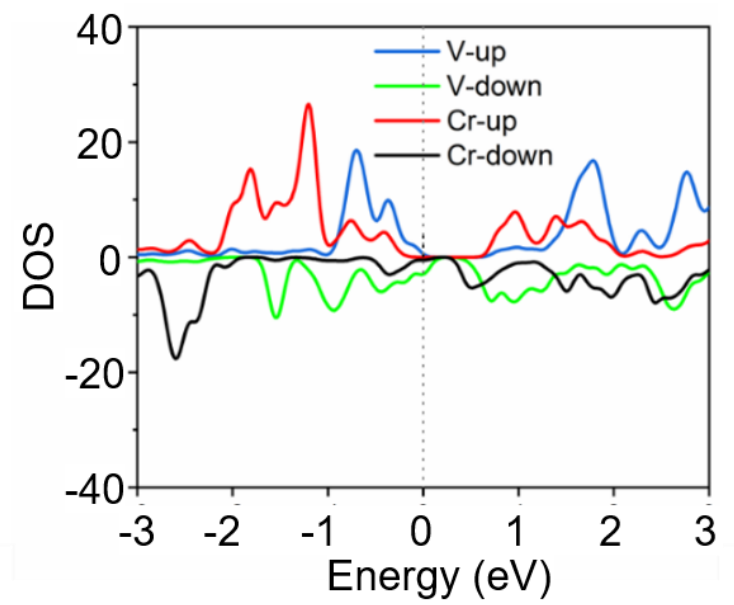

b

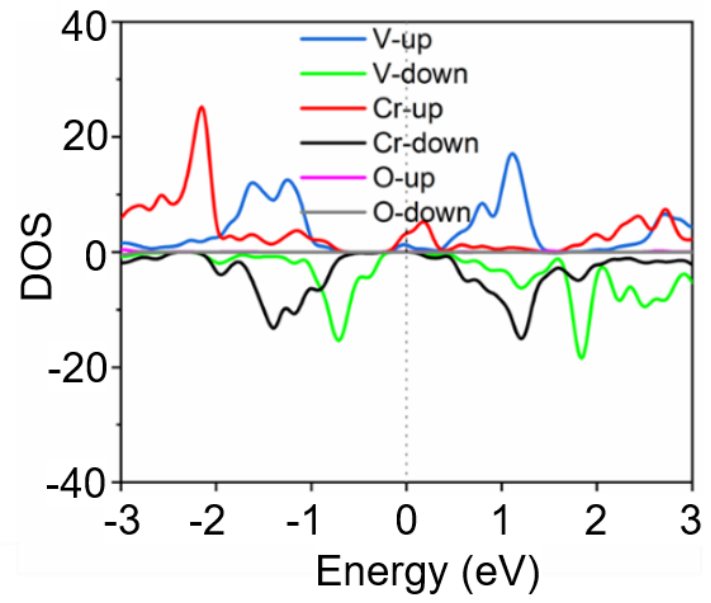

Figure S11. Density of states (DOS) of (a) $\left[\mathrm{VCr}(\mathrm{CN})_{6}\right](001)$ surface, (b) $\left[\mathrm{VCr}(\mathrm{CN})_{6}\right](001)$ PSS interface. The Fermi level is set to zero as shown by the dashed line. 


\section{Conductivities and hopping mode fitting of MCs.}

Temperature dependent conductivity is measured from $300 \mathrm{~K}$ to $10 \mathrm{~K}$ for all those MCs. However, due to the insulating nature of pure PBA, some MCs cannot present a detectable conductivity if the concentration of PEDOT:PSS is too low $(p<0.16)$. For the MC with $\mathrm{p}$ $=0.07$, a considerable conductivity can be measured only at the temperature range of above $215 \mathrm{~K}$ below which the conductivity is too small to be detected. All those MCs $(p>0.16)$ maintain a semiconducting behavior and a very low conductivity at $10 \mathrm{~K}$ due to carrier localization. MCs are regarded as electron disordered systems even if the local structural order exist in PBA and PEDOT:PSS in a short range. Thus, electron hopping mode is considered to describe the conductivity in cross-linking MCs.

The cross-linking MCs exhibit an enhanced electrical conductivity and tunable conducting mechanism. The electron carriers in $\mathrm{V}-\mathrm{Cr}$ PBA are localized with a limited proton conductivity ${ }^{[8,9]}$. By cross-linking with PEDOT:PSS polymers, MCs present the considerably enhanced electric conductivity, eventhough still remain a semiconducting behavior at low temperature. A kink behavior at $T^{*} \sim 150 \mathrm{~K}$ indicates that PEDOT:PSS not only increases the conductivity of MCs, but also controls its conducting switch through cross-linking ${ }^{[10]}$. With increasing the concentration of PEDOT:PSS, the kink temperature $T^{*}$ increases from $150 \mathrm{~K}$ to $200 \mathrm{~K}$. The variable range hopping theory ${ }^{[11]}(\boldsymbol{\sigma}(\boldsymbol{T})=$ $\sigma_{0} \exp \left[-\left(\frac{T_{0}}{T}\right)^{\frac{1}{n+1}}\right]$, where $\left.\mathrm{n}=0,1,2,3\right)$ is used to describe the conducting behavior in MCs (Figures 5b and S5-S8). The conductivities of MCs ( $p=0.16,0.37$ and 1.21) can be well fitted by three-dimensional variable range hopping model $(n=3)$. Light illumination induces an enhanced electrical conductivity in MCs (Figure S9), which may result from the charge transfer in PEDOT:PSS and its cross-linking with PBA. 

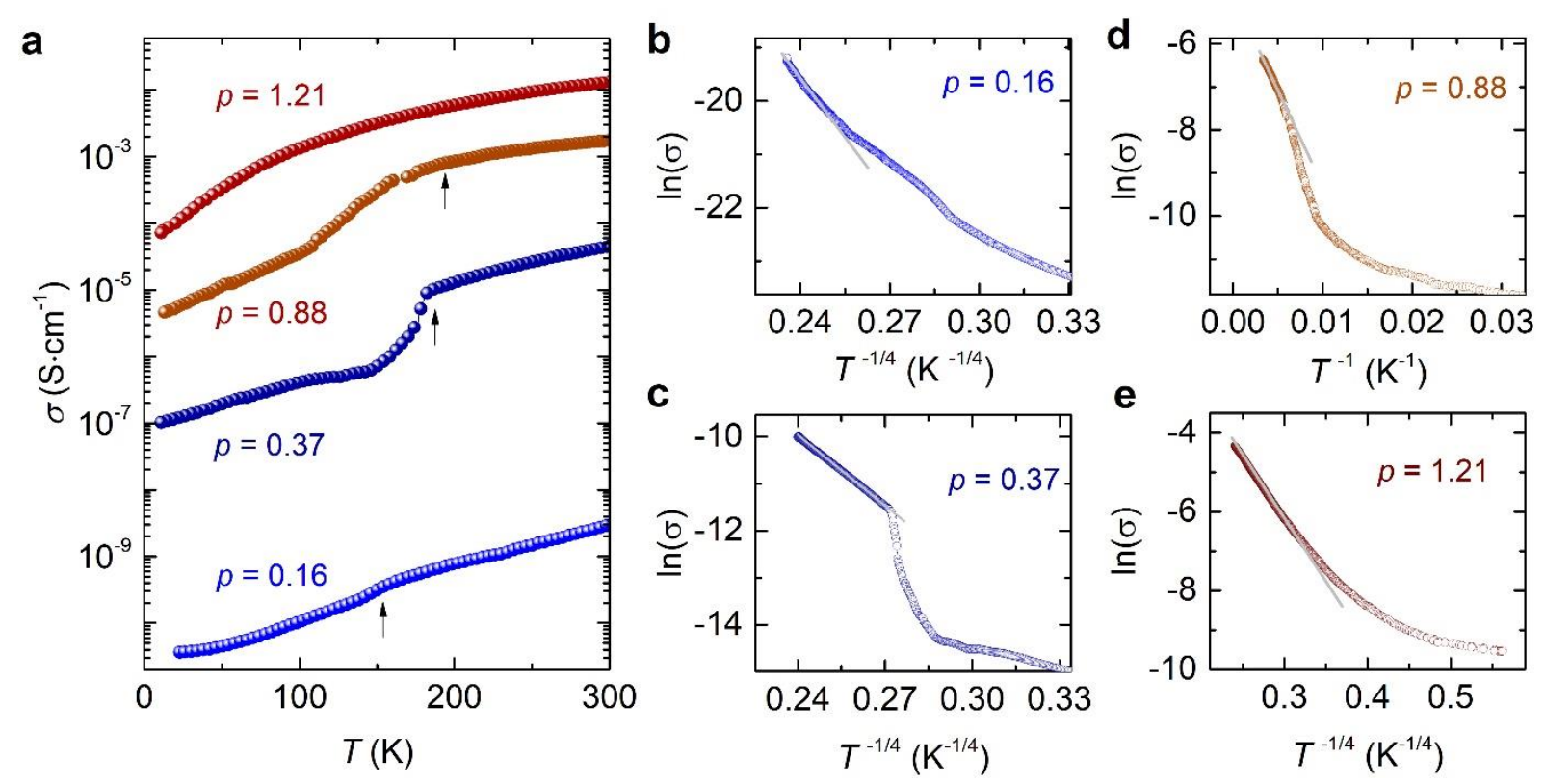

Figure S12. Low-temperature electrical conductivities of MCs from $300 \mathrm{~K}$ to $10 \mathrm{~K}$. (a) Temperature dependent conductivities of MCs with $p$ from 0.16 to 1.21 show an enhanced conductivity due to the increasment of PEDOT:PSS concentration. Characteristic temperature $150 \mathrm{~K}$ indicates conducting carriers proceed a localization transtion where carrier density or mobility is reduced. At $p=0.37$, the localization transition is more pronounced at $182 \mathrm{~K}$. A much broad transition at $200 \mathrm{~K}$ occurs in MCs of $p=0.88$. At a higher concentration of PEDOT:PSS ( $p=1.21$ ), the electrical transport behavior is dominant by PEDOT:PSS phase with an electrical conductivity of $9.3 \mathrm{mS} \cdot \mathrm{cm}^{-1}$ at $300 \mathrm{~K}$. (b-e) Carrier hopping model fittings of the electrical conductivities before the transition indicate three-dimensional variable-range hopping conductance in all MCs except for $p=0.88$ where nearest neighbor hopping is dominant. 

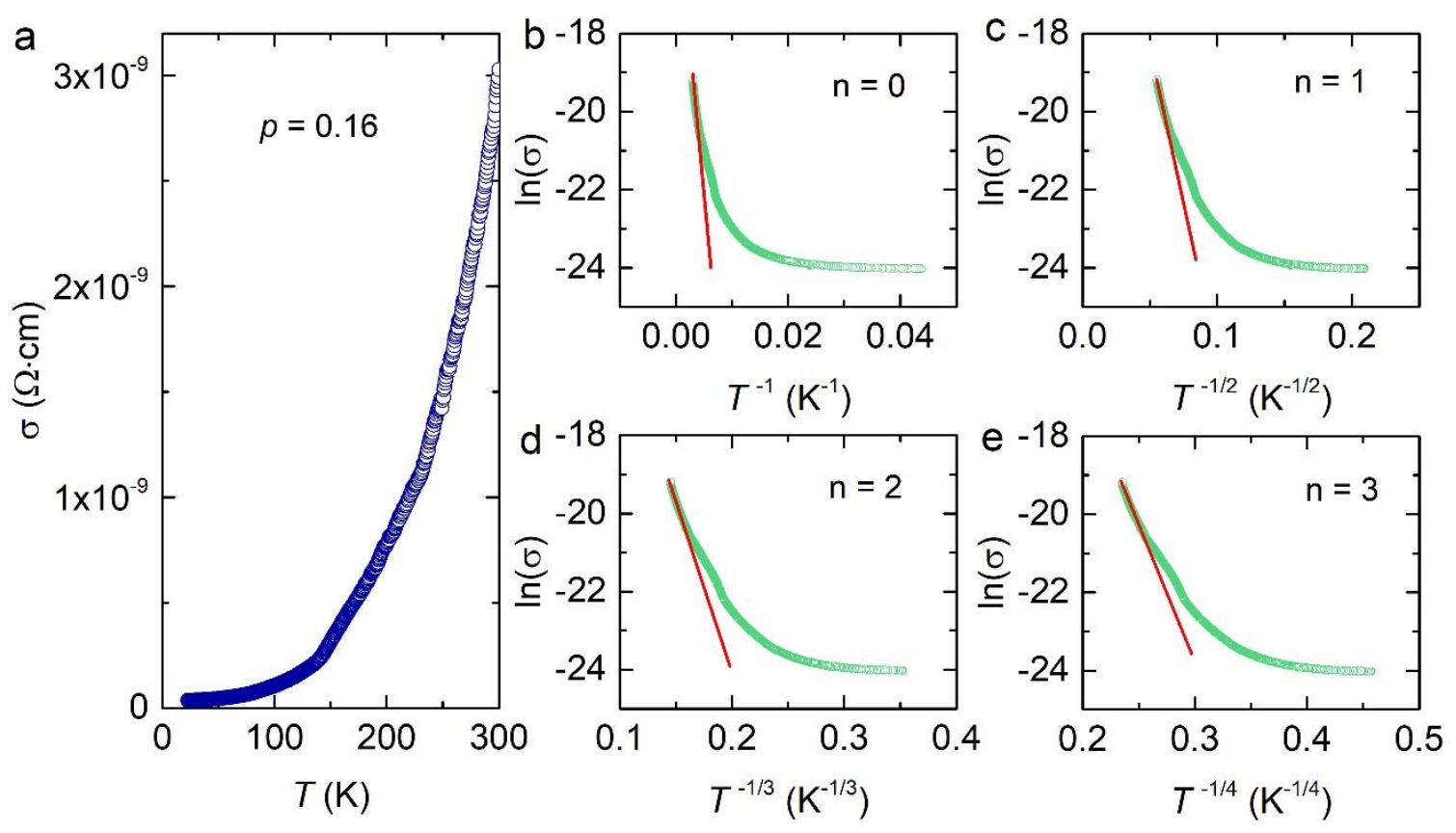

Figure S13. Conductivity of MC with $\mathrm{p}=0.16$. (a) Temperature dependent conductivity from $10 \mathrm{~K}$ to $300 \mathrm{~K}$; Fitting by conductivity hopping model for (b) $n=0$, (c) $n=1$, (d) n =2, (e) n $=3$. 

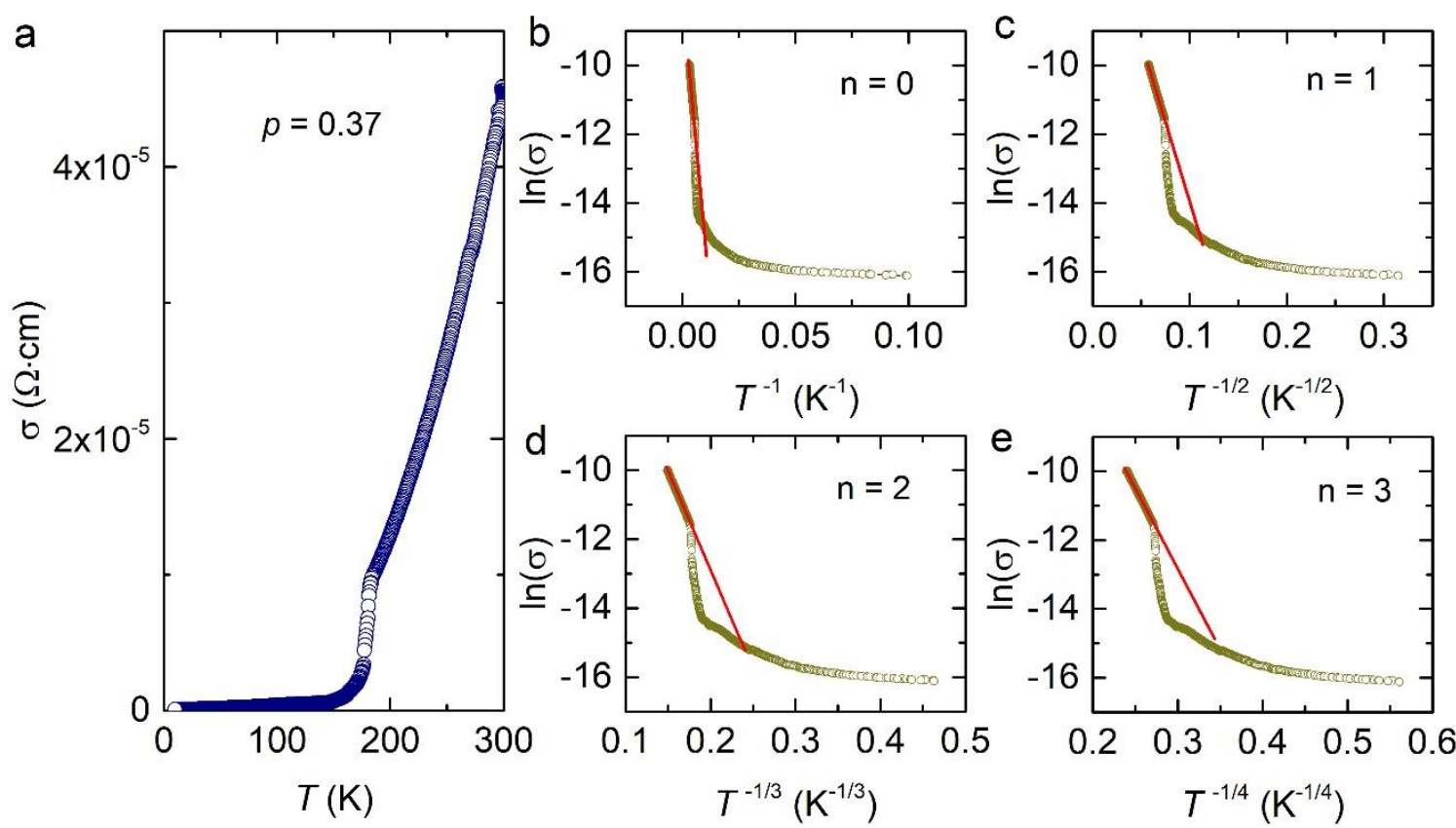

Figure S14. Conductivity of MC with $\mathrm{p}=0.37$. (a) Temperature dependent conductivity from $10 \mathrm{~K}$ to $300 \mathrm{~K}$; Fitting by conductivity hopping model for (b) $n=0$, (c) $n=1$, (d) $n=2$, (e) $n=3$. 

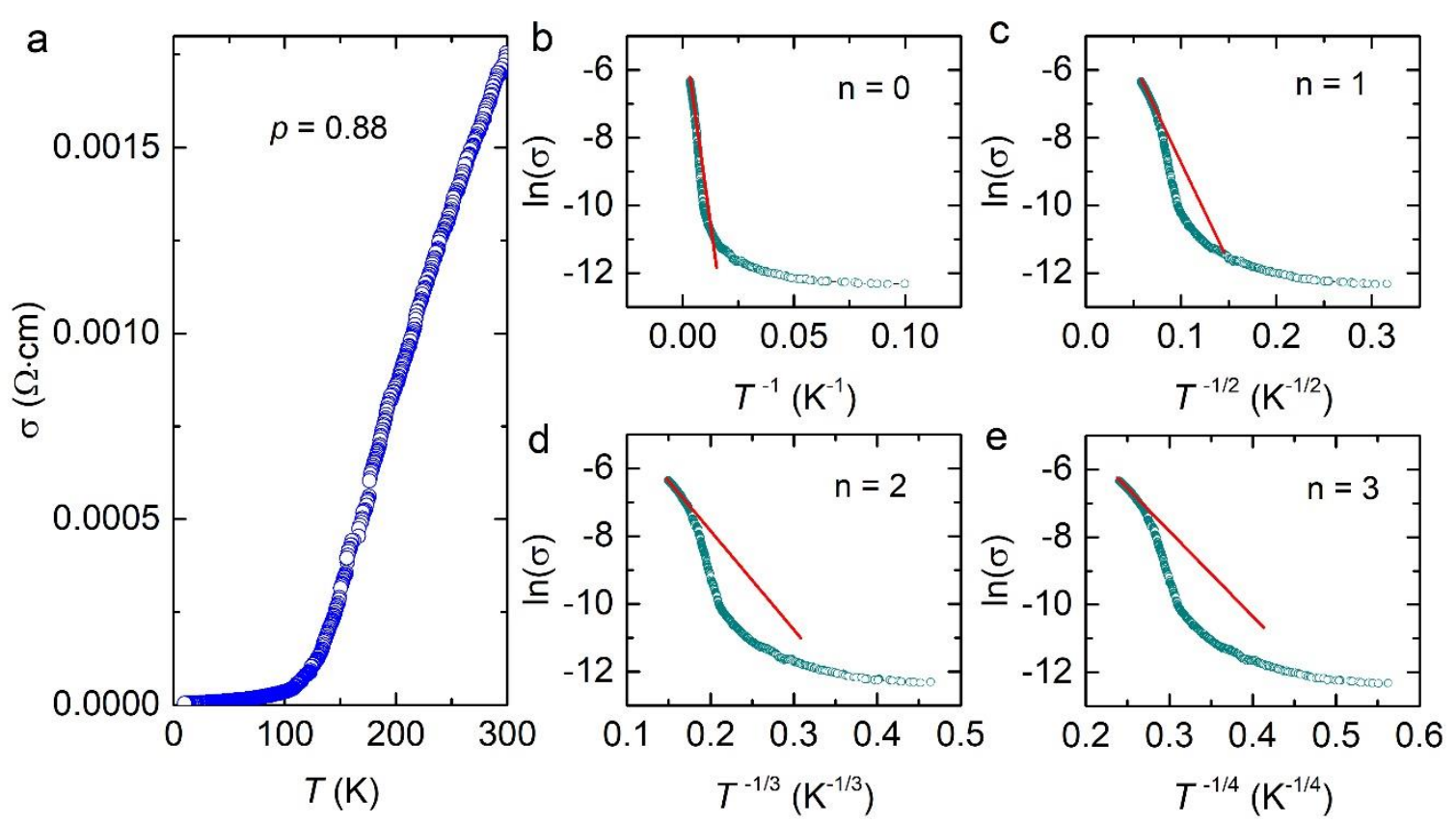

Figure S15. Conductivity of MC with $\mathrm{p}=0.88$. (a) Temperature dependent conductivity from $10 \mathrm{~K}$ to $300 \mathrm{~K}$; Fitting by conductivity hopping model for (b) $n=0$, (c) $n=1$, (d) $n=2$, (e) $n=3$. 

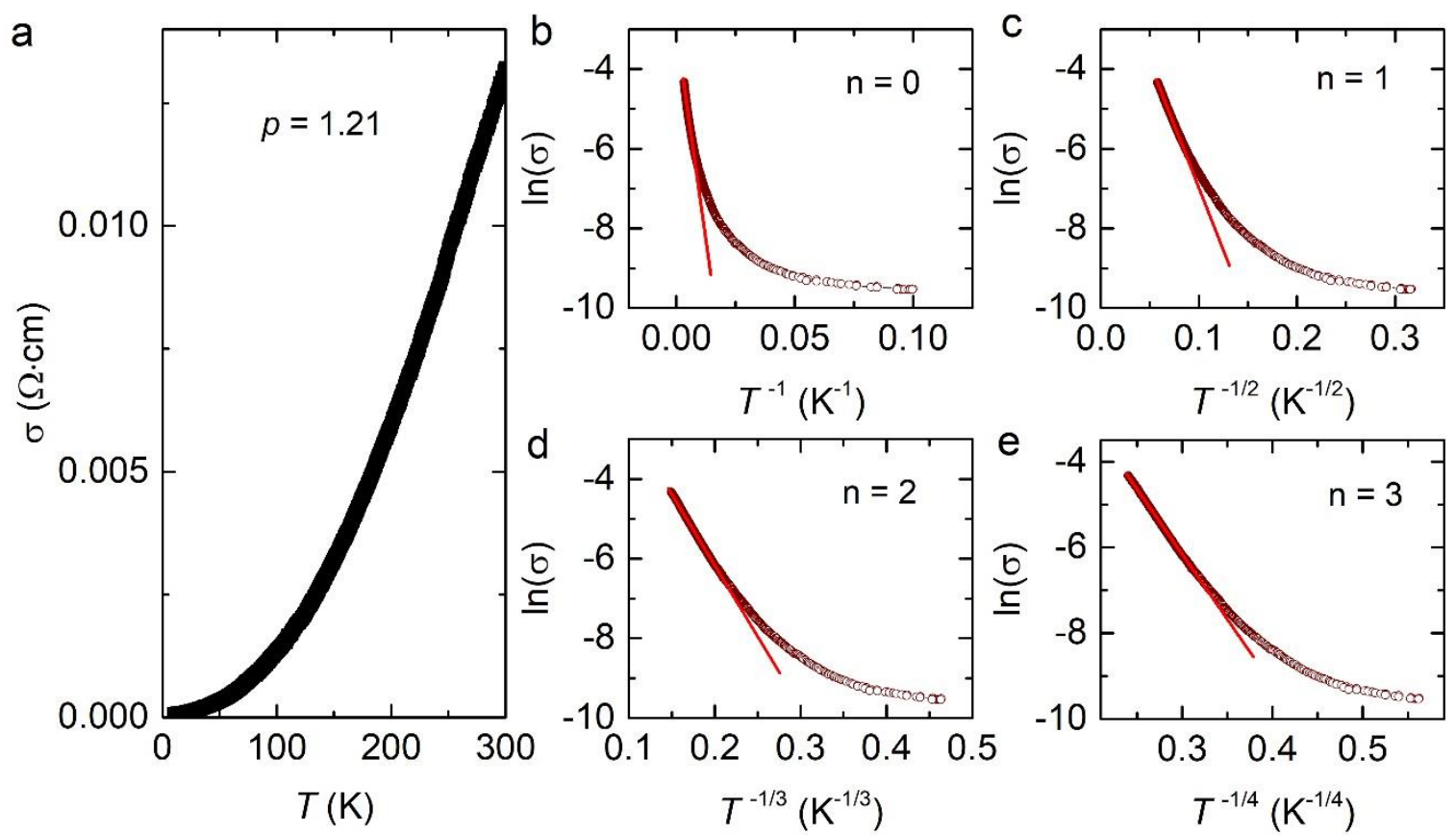

Figure S16. Conductivity of $\mathrm{MC}$ with $\mathrm{p}=1.21$. (a) Temperature dependent conductivity from $10 \mathrm{~K}$ to $300 \mathrm{~K}$; Fitting by conductivity hopping model for (b) $n=0$, (c) $n=1$, (d) n=2, (e) $n=3$. 


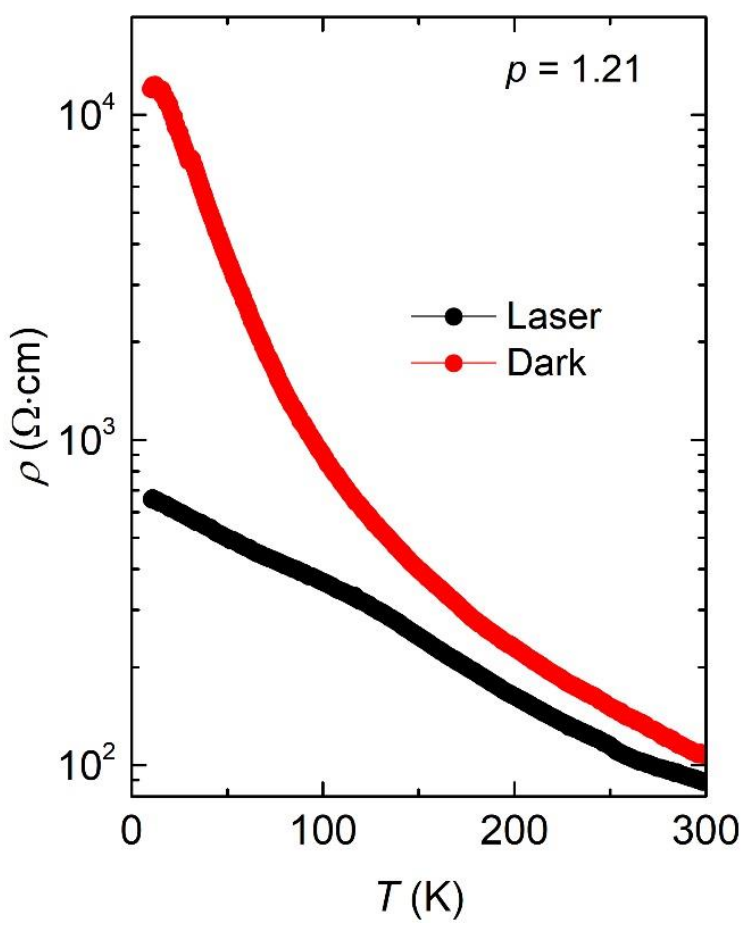

Figure S17. Temperature dependent resistivity of MC with $p=1.21$ under dark and laser $(\lambda=$ $405 \mathrm{~nm})$. 


\section{Absorbance spectra of MCs under electric field.}

The absorbance spectra for charge transfer in $\mathrm{Cr}^{\mathrm{II}} \mathrm{C} \equiv \mathrm{NV}^{\mathrm{II}}$ around $560 \mathrm{~nm}$ and a forbidden transition in $\left[\mathrm{Cr}(\mathrm{CN})_{6}\right]^{3-}$ around $620 \mathrm{~nm}$ are studied under electric field. The absorbance magnitude is decreasing by increasing electric field, implying molecular absorbance is influenced.
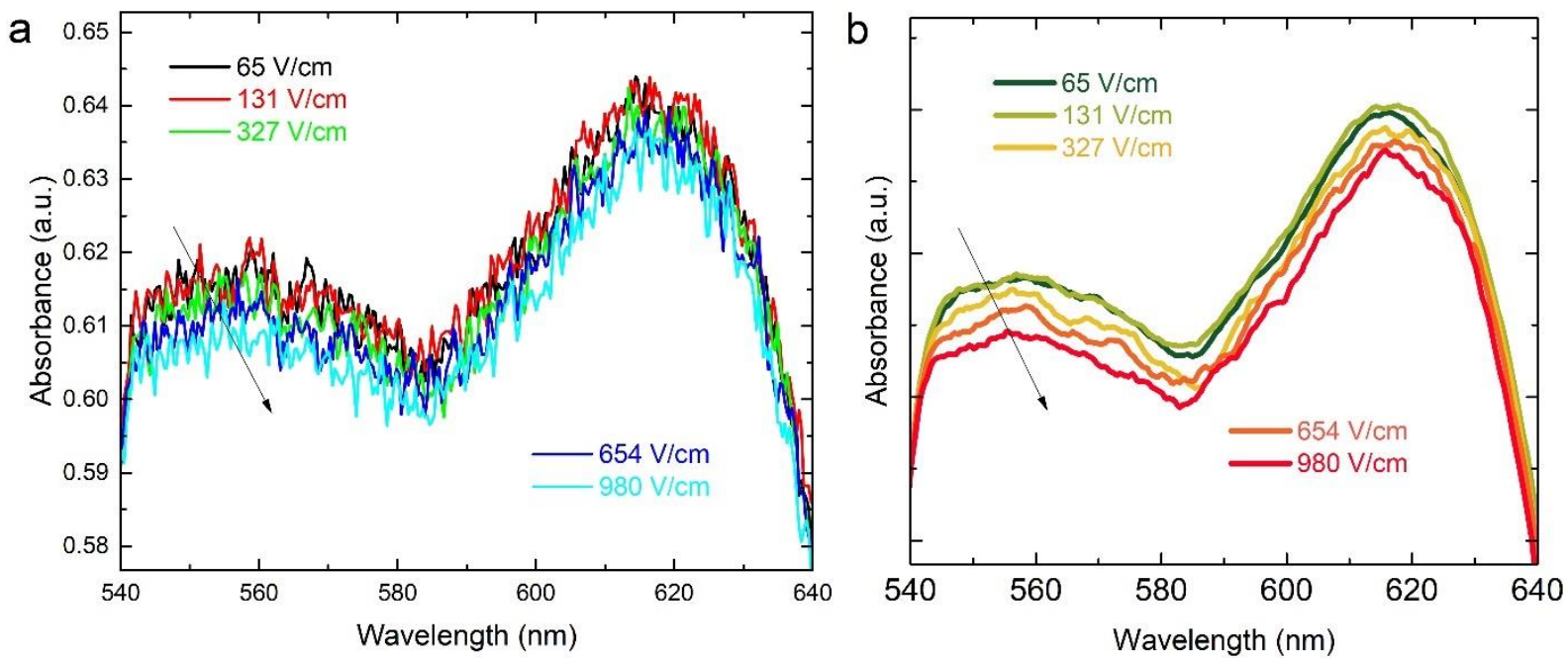

Figure S18. Absorbance spectra of MC under electric field. (a) The raw data of absorbance spectra of MC were taken under increasing electric fields. (b) The smooth results by using an adjacent-averaging method. 


\section{Temperature dependent magnetization of pure PBA under electric field.}

Electric field is applied to study magneto-electronic coupling in MCs whose magnetization can be controlled. As a reference, the pure PBA shows no electric-field effects.

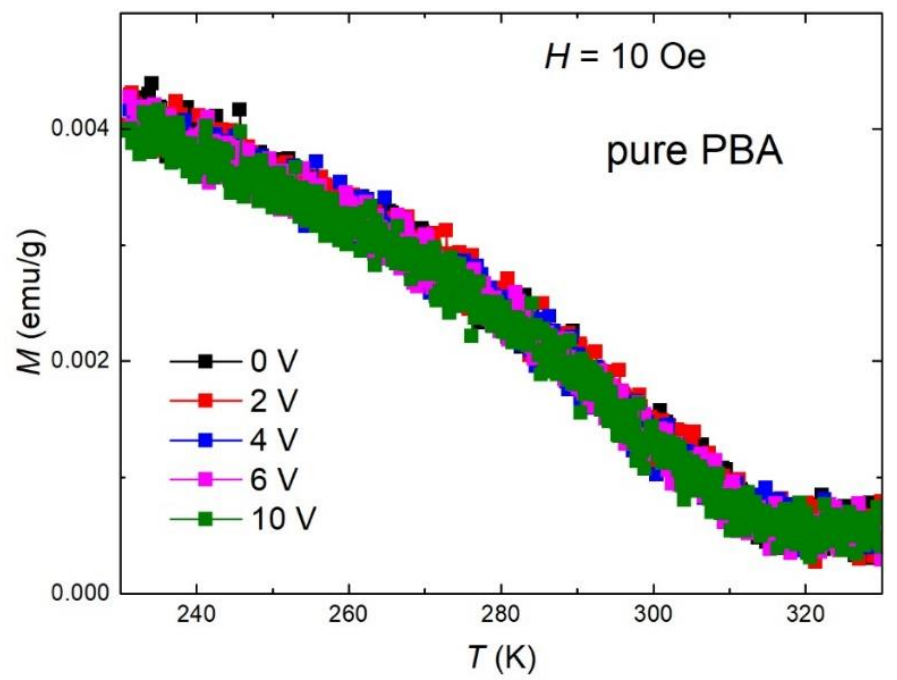

Figure S19. Temperature dependent magnetization of PBA under electric field.

\section{References:}

[1] G. Kresse, J. Furthmüller, Computational Materials Science 1996, 6, 15.

[2] G. Kresse, J. Furthmüller, Phys. Rev. B 1996, 54, 11169.

[3] J. P. Perdew, K. Burke, M. Ernzerhof, Phys. Rev. Lett. 1996, 77, 3865.

[4] S. Grimme, J. Antony, S. Ehrlich, H. Krieg, The Journal of Chemical Physics 2010, 132, 154104.

[5] V. I. Anisimov, J. Zaanen, O. K. Andersen, Phys. Rev. B 1991, 44, 943.

[6] Vadym V. Kulish, S. Manzhos, RSC Advances 2017, 7, 18643.

[7] P. Hejduk, M. Witko, K. Hermann, Topics in Catalysis 2009, 52, 1105.

[8] S. Ohkoshi, K. Nakagawa, K. Tomono, K. Imoto, Y. Tsunobuchi, H. Tokoro, J Am Chem Soc 2010, 132, 6620.

[9] X. Wu, J. J. Hong, W. Shin, L. Ma, T. Liu, X. Bi, Y. Yuan, Y. Qi, T. W. Surta, W. Huang, J. Neuefeind, T. Wu, P. A. Greaney, J. Lu, X. Ji, Nature Energy 2019, 4, 123. [10] L. Yan, X. Gao, J. P. Thomas, J. Ngai, H. Altounian, K. T. Leung, Y. Meng, Y. Li, Sustainable Energy \& Fuels 2018, 2, 1574.

[11] B. I. Shklovskii, A. L. Efros, in Electronic Properties of Doped Semiconductors, 1984, 202. 